\title{
No Effect of Transcranial Direct Current Stimulation on Anaerobic Test Performance in Resistance- Trained Individuals
}

Original Research

\author{
Nicholas J. Hanson ${ }^{1}$, Rachel M. Dykstra ${ }^{2} \&$ Kyle D. DeRosia ${ }^{3}$ \\ ${ }^{1}$ Department of Human Performance \& Health Education, Western Michigan University, Kalamazoo, MI/USA \\ ${ }^{2}$ Department of Health \& Human Performance, Nebraska Wesleyan University, Lincoln, NE/USA \\ ${ }^{3}$ Department of Public Health \& Human Sciences, Oregon State University, Corvallis, OR/USA
}

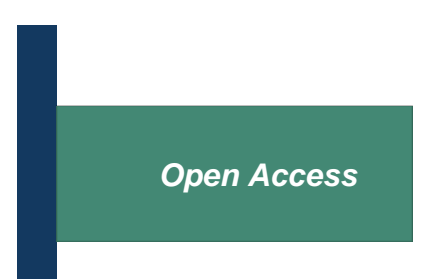

Published: August 9, 2021

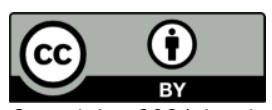
authors. Published by Research Directs and the work is licensed under the Creative Commons Attribution 4.0 International License. To view a copy of this license, visit http://creativecommons .org/licenses/by/4.0/

Research Directs in Strength and

Performance: 2021,

Volume 1 (Issue 1): 6
Copyright, 2021 by the

ISSN: 2768-5187

\begin{abstract}
Introduction: Transcranial direct current stimulation (tDCS) sends a weak electrical current through the cerebral cortex. tDCS has been shown to be effective in longer activities $(>75 \mathrm{~s})$ but minimal research has been performed with short, anaerobic tests. The purpose of this study was to determine the effect of tDCS on Wingate Anaerobic Test (WAnT) performance.

Methods: Fifteen young, resistance-trained adults $(23.7 \pm 2.7$ years; BMI $24.9 \pm 2.6$ $\mathrm{kg} \cdot \mathrm{m}^{-2} ; 12$ males) volunteered for this study. Electrodes were placed at T3 and FP2 for anodal stimulation of the insular cortex (IC), and $2 \mathrm{~mA}$ of current was supplied for 20 minutes; after a short rest period, subjects performed a WAnT. Dependent variables included peak/mean/relative power, peak heart rate (HR) and rating of perceived exertion (RPE). Experimental and sham conditions were utilized. Pairedsamples t-tests were used to determine the effect of tDCS on the dependent variables.

Results: Peak power in the experimental condition $(1,019.0 \pm 237.5 \mathrm{~W})$ was not different than that of the sham $(1,008.3 \pm 240.4 \mathrm{~W} ; \mathrm{p}=.638)$. There were no differences in any other WAn'T variables, and no differences in peak HR or RPE (all p>.05).

Conclusions: The results from this study suggest that tDCS in resistance-trained individuals is not effective in improving performance on an anaerobic test. In addition, it is still considered experimental and its ethical use is questionable.
\end{abstract}

Key Words: neurodoping, insular cortex, Wingate

Corresponding author: Nicholas J Hanson, njhanson@gmail.com

\section{Introduction}

Transcranial direct current stimulation (tDCS) is a non-invasive technique in which a weak electrical current of $\sim 1-2 \mathrm{~mA}$ is sent through the cerebral cortex using electrodes placed on the scalp. This small amount of current, roughly equivalent to that of a 9-volt battery, is believed to increase excitability of the underlying neurons in the stimulated area by altering the resting membrane potential ${ }^{1}$. The specific placement of tDCS electrodes, typically left on a subject for 10-30 minutes, is based on the desired outcome ${ }^{2}$. According to a recent review on the safety of tDCS, any adverse effects are very minimal and usually limited to a mild tingling sensation at the location of the electrodes, moderate fatigue, or itching 3,4 . The notion of applying electricity to the scalp to introduce changes in the brain is by no means a new concept and has been used for $100+$ years ${ }^{5}$. However, in the past 20 years it has been used extensively with various populations and for many different 
purposes. For example, tDCS is commonly used for treatment of depression ${ }^{6}$, enhancement of working memory ${ }^{7}$, or for accelerated learning within a military setting ${ }^{8}$.

The use of tDCS in an attempt to improve sport performance has increased in recent years. Studies normally use anodal stimulation and implement a "sham" treatment where the subject does not actually receive stimulation. Multiple studies have shown an improvement in performance with tDCS stimulation over the motor cortex (M1). This area of the brain is most directly related to exercise performance as it is responsible for neural drive to the muscles ${ }^{9}$. Angius et al. ${ }^{10}$ and Vitor-Costa et al. ${ }^{11}$ showed marked improvements in time to exhaustion (TTE) tests after M1 stimulation. Similarly, Williams et al. ${ }^{12}$ reported improved time to task failure during a sustained submaximal contraction. Sasada et al. ${ }^{13}$ had subjects complete a 30s maximal cycling test and found greater mean power output. Another brain region that has the potential to improve athletic performance is the insular cortex (IC), which has been shown to be involved with emotions, specifically with the processing of pleasant sensations. The IC is also responsible for parasympathetic modulation at rest and during exercise ${ }^{14}$ as well as self-awareness and involvement with bodily movements ${ }^{15}$. Only two exercise-based studies could be located that used the IC as the target region during tDCS, and the results are equivocal. Okano et al. ${ }^{1}$ reported increased peak power output in a cycling test with no change in maximal ratings of perceived exertion (RPE). Conversely, Barwood et al. ${ }^{16}$ showed no improvement in mean power output during a cycling time trial, and no change in TTE or RPE.

tDCS has been shown to be effective in aerobic activities but thus far very minimal research has been performed with short, anaerobic tests. In fact, a recent review on the effect of tDCS on exercise performance only included activities lasting greater than 75 seconds ${ }^{2}$. More information needs to be gathered on the efficacy of tDCS in relation to improving performance within short-term activities where high levels of power production are critical such as the Wingate Anaerobic Test (WAn'T) ${ }^{17}$.

The main purpose of this study was to examine the effect of tDCS on anaerobic test performance in resistance-trained individuals. Our hypothesis was that compared to a sham condition, where subjects are led to believe that they are receiving treatment but are not, experimental tDCS would lead to increased peak power output during an anaerobic test with no difference between conditions in peak RPE.

\section{Scientific Methods}

Subjects

Fifteen adult subjects $\left(23.7 \pm 2.7\right.$ years; BMI $24.9 \pm 2.6 \mathrm{~kg} \cdot \mathrm{m}^{-2} ; 12$ males $)$ volunteered for this study, which was approved by the university's Human Subject Institutional Review Board. All subjects read and signed an informed consent form prior to participation. Exclusion criteria included any history of epilepsy, seizures, or lower body musculoskeletal injury within the last six months. Participants were considered low risk (less than two cardiovascular risk factors) based on the American College of Sports Medicine health screening ${ }^{18}$. All subjects identified themselves as resistance-trained individuals and were primarily involved with strength and power-related activities.

\section{Protocol}

Subjects completed experimental and sham conditions, which were both single blinded. They were required to complete the two laboratory visits within one week with at least 24 hours between each visit; the order was counterbalanced. After reading and signing the informed consent form, they were then familiarized with the equipment and procedures that would be used. Subjects' height, weight, and age were then recorded and the Borg 6-20 RPE scale ${ }^{19}$ was explained in detail. Heart rate (HR) was monitored continuously from the start of the laboratory session to the end of the cool down using a Garmin Forerunner 630 (Garmin, Inc.; Olathe, Kansas). When preparing to position and administer the tDCS treatment, subjects were asked to sit upright in a reclining dental chair in an isolated room to help mitigate distractions. Alcohol wipes were used to clean the electrode sites for proper conduction. $2 \times 2$ inch sponge electrodes soaked with a $0.9 \%$ saline solution were used at the electrode sites, which were T3 (anode) and FP2 (cathode) according to the international EEG 10-20 standards ${ }^{20}$. This placement has been used previously to direct the current through the left IC ${ }^{14}$. In the experimental condition, there was a $30 \mathrm{~s}$ ramp up to $2 \mathrm{~mA}$ which was held constant through the trial, and then ramped down during the final 30s. The sham condition was identical to the experimental condition except for the delivery of electrical stimulation. Similar to the technique suggested previously ${ }^{21}$, the current was ramped up to $2 \mathrm{~mA}$ over $30 \mathrm{~s}$ and then immediately ramped down, at the beginning and again at the end of the 20 minute period (i.e., at 0:00 and 19:00, there was a ramp up to $2 \mathrm{~mA}$ and at 0:30 and 19:30 there was a ramp down to $0 \mathrm{~mA}$ ). This method has been shown to elicit similar responses to continuous electrical stimulation (itching, tingling, etc.) and 
subjects often report lingering sensations following the termination of stimulus. This allowed the subjects to be blinded to which condition was taking place.

Following completion of the 20-minute experimental tDCS or sham treatment, subjects completed a 5-minute warmup on a cycle ergometer. This warm-up was self-paced but consisted of the subjects sprinting with maximal effort for the last 5 seconds of every minute. Within 5 minutes of completing the warm-up, the subjects were asked to complete the WAnT on a Monark cycle ergometer. This time frame allowed consistent testing completion within 10 minutes past the end of the stimulation. The WAnT protocol consisted of a flywheel resistance of 9\% body mass. Studies have suggested that higher braking forces are optimal for active subjects compared to the traditional $7.5 \%$ body mass 22 . Once subjects reached a pedal frequency of 120 revolutions per minute, the weight basket dropped onto the flywheel and the 30s timer began. The variables collected by the Monark software included peak/mean power, time to peak power, relative peak/mean power, peak cadence and fatigue index. Immediately following completion of the WAnT, subjects were asked to report their RPE. Afterward, the subjects were allowed to cool down by either walking around the laboratory or continuing to cycle on the ergometer with no resistance.

Statistical Analysis

Data were analyzed using SPSS (Version 25; IBM, Armonk, NY). Paired samples t-tests were used to determine the effect of tDCS condition (experimental or sham) on the dependent variables. Significance level was set a priori at $\mathrm{p}<.05$. Data is presented as means \pm standard deviations. Effect sizes are presented as Cohen's $d$.

\section{Results}

There was no improvement in peak power after the experimental tDCS treatment compared to the sham (1024.2 \pm 245.6 vs. $1012.4 \pm 248.9 \mathrm{~W}$, respectively; $\mathrm{p}=.632,95 \%$ CI $[-40.1,63.7])$. There were also no differences in relative peak or mean power, time to peak power, fatigue index, peak cadence, HR or RPE (Table 1). The pacing strategy between conditions was very similar and can be seen in Figure 1, which shows power output each second during both tests. Figure 2 shows the peak power output for each subject in both conditions.

Table 1. Comparison of variables between experimental and sham conditions.

\begin{tabular}{|c|c|c|c|c|}
\hline & EXPERIMENTAL & SHAM & $\mathrm{p}$ value & Effect size \\
\hline Peak Power (W) & $1,019.0 \pm 237.5$ & $1,008.3 \pm 240.4$ & .638 & .123 \\
\hline Rel. Peak Power $\left(W \cdot \mathbf{k g}^{-1}\right)$ & $13.4 \pm 1.8$ & $13.2 \pm 1.8$ & .656 & .180 \\
\hline Time to Peak Power (ms) & $2,276.1 \pm 1534.4$ & $1,900.9 \pm 840.1$ & .418 & .215 \\
\hline Mean Power (W) & $707.0 \pm 166.5$ & $702.8 \pm 165.4$ & .599 & .137 \\
\hline Rel. Mean Power $\left(\mathrm{W} \cdot \mathrm{kg}^{-1}\right)$ & $9.3 \pm 1.1$ & $9.2 \pm 1.1$ & .587 & .267 \\
\hline Fatigue Index (\%) & $57.6 \pm 7.1$ & $58.1 \pm 8.4$ & .779 & .076 \\
\hline Peak Cadence (rpm) & $143.3 \pm 13.0$ & $144.0 \pm 12.0$ & .803 & .070 \\
\hline Peak heart rate (bpm) & $183.5 \pm 8.1$ & $182.4 \pm 9.8$ & .468 & .205 \\
\hline RPE & $18.3 \pm 1.6$ & $17.7 \pm 1.9$ & .057 & .539 \\
\hline
\end{tabular}

Note: Values are given as means \pm standard error. $\mathrm{W}=$ Watts, $\mathrm{W} \cdot \mathrm{kg}^{-1}=\mathrm{Watts}$ per Kilogram. Effect sizes are presented as Cohen's $d$ (small: 0.2, medium: 0.5, large: 0.8) ${ }^{23}$. 


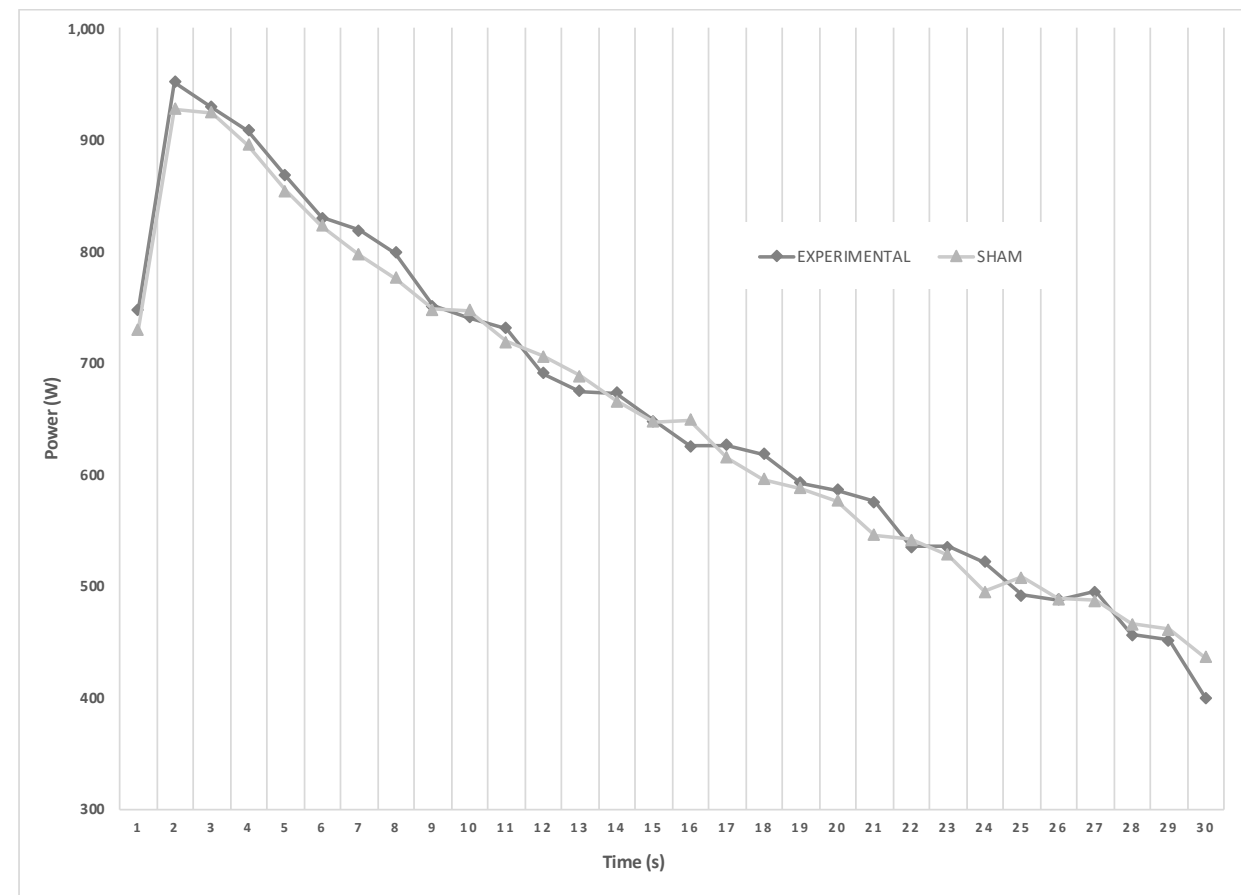

Figure 1. Mean power output during WAnT testing, assessed every second; no significant differences (all $\mathrm{p}>.05$ ) between conditions for any time point.

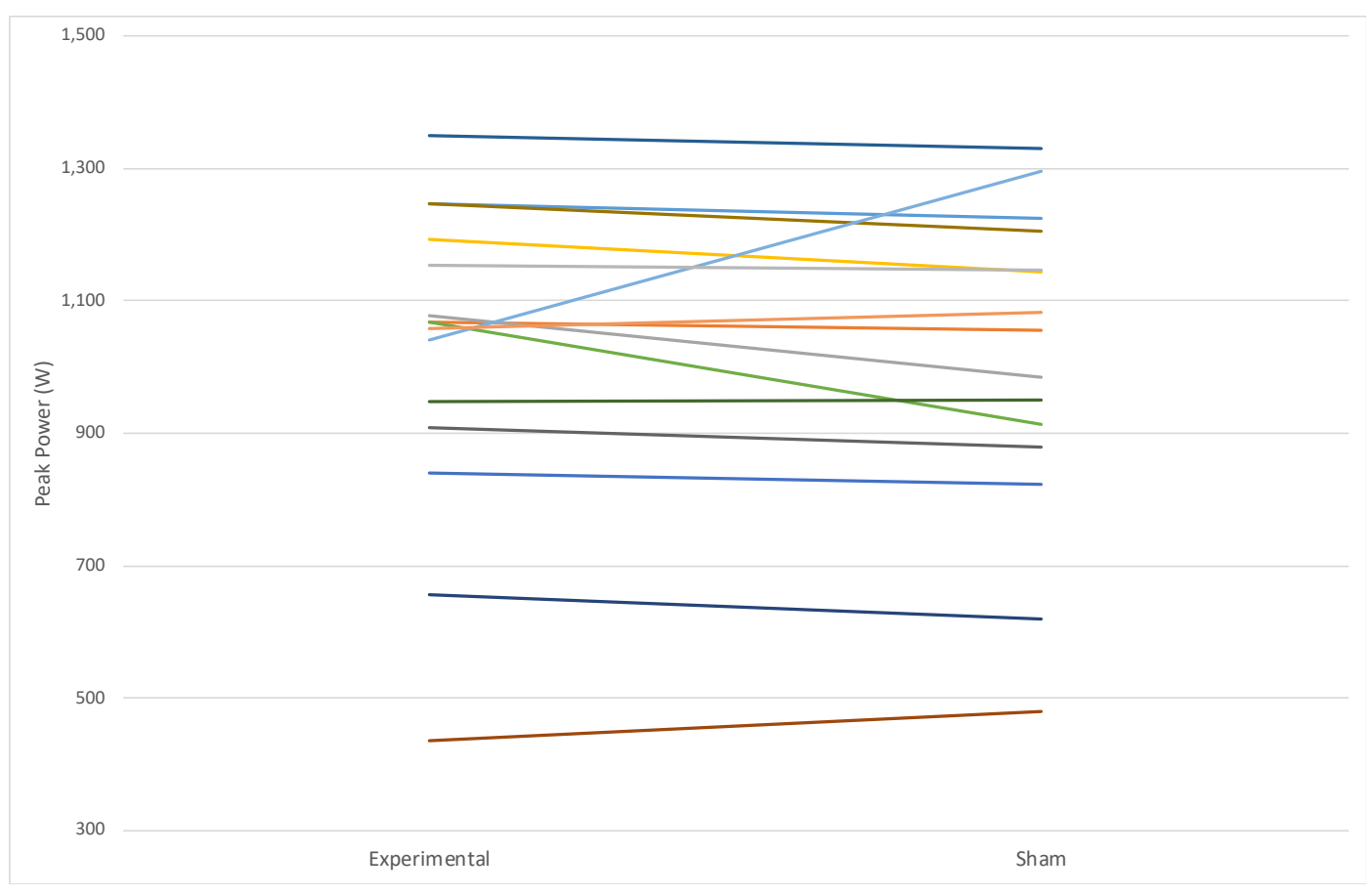

Figure 2. Peak power output for each subject. 


\section{Discussion}

The main finding from the present study was that tDCS over the insular cortex was unable to elicit improvements in power output during a short, anaerobic test in resistance-trained individuals. Additionally, no changes in any of the other WAnT variables, HR or RPE were found between the experimental and sham conditions. Therefore, our hypothesis was not supported. Many studies have shown that tDCS is able to provide an improvement in performance; however, there are just as many studies showing no difference between tDCS and a sham condition. Determining the reasons behind these differences is somewhat difficult. It is possible that contradictory findings within previous studies using tDCS may be related to differences in methodology, such as electrode placement or chosen exercise protocol.

There are many electrode configurations and brain regions that researchers have targeted ${ }^{9}$. Stimulation of the motor cortex is the most common; it is most directly related to exercise performance due to its major role in providing neural drive to working skeletal muscle ${ }^{24}$. There is justification to use tDCS over this region as it could increase the extent of neural activity, which could improve power and increase time to fatigue. In addition, it has been shown that stimulation over this region has the ability to inhibit the perception of pain ${ }^{25}$. Another brain region that has been used with tDCS is the prefrontal cortex, which plays a major role in processing cues (both internal and external) related to exercise ${ }^{26}$. Stimulation of this region may have the ability to positively affect variables surrounding exercise performance such as decision-making, effort perception, endpoint knowledge and mental fatigue ${ }^{27}$. In the present study the IC was chosen as the region of interest due to the large part that it plays in emotions, pleasant sensations, and subjective body feelings 1. Individuals experienced with resistance training were chosen for participation; the rationale was that these subjects were familiar with exercise utilizing the two energy pathways involved with the WAnT (ATP-PCr and anaerobic glycolysis). Previous research has shown that subjects perform best during exercise/sports in which they are very familiar, suggesting that stimulation of the IC would increase positive emotions surrounding said exercise. This increase in positive emotions, then, would lead to a greater performance (i.e., power output) during the exercise. The findings did not support this hypothesis, however, as no improvements in any of the WAnT variables was shown.

The exercise protocol may affect the ability of tDCS to improve performance as well. Previous studies range from testing isometric, isokinetic, and dynamic strength, to various forms of cycling exercise ${ }^{9}$. In the present study, the chosen protocol was the WAnT, a test of anaerobic power and capacity ${ }^{28}$ that is used in numerous sports at all levels. The WAnT is a high intensity anaerobic test that is "supramaximal," meaning that it requires a work rate that is $\sim 2-4$ times greater than one's maximal aerobic power ${ }^{17}$. The main variables obtained from this test are peak power, average power, and fatigue index. Peak power, a measure of anaerobic power that is an instantaneous product of force/velocity ${ }^{29}$, is typically reached in the first few seconds of the test when the subject is at maximal velocity. The average power output over the entire test is known as the anaerobic capacity. Fatigue index, or "power drop," is the difference between the peak power and the lowest recorded power output. These variables were not improved with tDCS over the IC in the present study. While stimulation of the IC has been shown to improve power output ${ }^{1}$, results from the present study suggest that it cannot improve performance on a maximal anaerobic test such as the WAnT. It is likely that subjects reached their physiologic limit during the WAnT, and any alterations in emotions or feelings was unable to allow the production of a greater power output.

Very few tDCS studies have used resistance-trained individuals as subjects. Lattari et al. ${ }^{30}$ had subjects experienced with resistance training complete elbow flexion exercise at 10RM load after anodal stimulation over the dorsolateral prefrontal cortex. They found increased repetitions completed compared to a sham condition, as well as a reduction in RPE. In a later study, the same research group also showed improved performance in the leg press $45^{\circ}$ (LP45) test and lower RPE utilizing the same electrode configuration and subjects familiar with resistance exercise ${ }^{31}$. Lattari et al. also used stimulation of M1 on individuals with advanced weight training experience and found a significant improvement in countermovement jump performance ${ }^{32}$. Multiple reviews $2,9,24,27,33$ have shown mixed but overall positive results with tDCS but there is a high likelihood of a publication bias toward studies with significant findings 33 as well as high inter-individual variability (i.e. responders/non-responders ${ }^{27}$ ). The present study displayed a relatively large amount of variability between subjects, as shown by Figure 2.

Despite conflicting results in controlled studies, it is worthwhile to mention a growing concern surrounding the ethical use of tDCS. The World Anti-Doping Association (WADA) has continuously made efforts to ban any substances or practices that take away the integrity, equity, and justice in sport. Explicitly, substances or practices that fall under two of the following three categories are banned from use in sport: drugs or tools that 1) secure a winning edge, 2) place athletes at health risk, or 3) ruin the spirit of sport. These will be banned from sport, but the process of elimination does not happen immediately. Delay of banning these substances or practices allows for loopholes in uses of ergogenic 
aids. Today, there are a collection of categories that have been permanently banned from sport (e.g. gene doping, blood components, and chemical or physical manipulations) ${ }^{34}$. Neuro-doping is not yet included, but because of its demonstrated performance enhancements in many studies, it is under consideration for review. A commercially available variant of tDCS that is built into a headphone unit, is available and is currently being utilized by multiple professional sports teams. The Halo Sport (Halo Neuroscience, CA) has been shown to improve jump force in ski jumpers by $70 \%$, in addition to an $80 \%$ increase in coordination after treatment ${ }^{35}$. However, research on this specific form of tDCS is limited and should be investigated in the future. As with all studies, there were certain limitations that should be mentioned. A full familiarization visit with a WAnT was not implemented, and this would have been beneficial to safeguard against any learning effect for those that had not performed the test prior to the study. Additionally, to reduce the number of visits required to the laboratory, there was no direct measure of fitness in this study. Future research studies should measure baseline strength or power in order to categorize participants in a way other than self-reported measures (e.g. novice and advanced).

\section{Conclusions}

Direct stimulation of the IC was unable to improve anaerobic power or capacity in resistance-trained individuals. Findings from this study and many others using tDCS question its ability to work in a consistent manner; in addition, it is still considered experimental and its ethical use is questionable. The authors do not suggest that strength and conditioning coaches or athletes utilize this method until more details are known about its effectiveness.

\section{References}

1. Okano AH, Fontes EB, Montenegro RA, et al. Brain stimulation modulates the autonomic nervous system, rating of perceived exertion and performance during maximal exercise. British journal of sports medicine. 2015;49(18):1213-1218.

2. Angius L, Hopker J, Mauger AR. The ergogenic effects of transcranial direct current stimulation on exercise performance. Frontiers in Physiology. 2017;8.

3. Poreisz C, Boros K, Antal A, Paulus W. Safety aspects of transcranial direct current stimulation concerning healthy subjects and patients. Brain Res Bull. 2007;72(4):208-214.

4. Reinhart RM, Cosman JD, Fukuda K, Woodman GF. Using transcranial direct-current stimulation (tDCS) to understand cognitive processing. Attention, Perception, \& Psychophysics. 2017;79(1):3-23.

5. Sarmiento C, San-Juan D, Prasath V. Letter to the Editor: Brief history of transcranial direct current stimulation (tDCS): from electric fishes to microcontrollers. Psychological Medicine. 2016;46(15):3259.

6. Loo CK, Sachdev P, Martin D, et al. A double-blind, sham-controlled trial of transcranial direct current stimulation for the treatment of depression. International Journal of Neuropsychopharmacology. 2010;13(1):61-69.

7. Fregni F, Boggio PS, Nitsche M, et al. Anodal transcranial direct current stimulation of prefrontal cortex enhances working memory. Experimental Brain Research. 2005;166(1):23-30.

8. Parasuraman R, McKinley RA. Using noninvasive brain stimulation to accelerate learning and enhance human performance. Human Factors. 2014;56(5):816-824.

9. Machado DGdS, Unal G, Andrade SM, et al. Effect of transcranial direct current stimulation on exercise performance: a systematic review and meta-analysis. Brain Stimulation. 2019.

10. Angius L, Hopker JG, Marcora SM, Mauger AR. The effect of transcranial direct current stimulation of the motor cortex on exercise-induced pain. European Journal of Applied Physiology. 2015;115(11):2311-2319.

11. Vitor-Costa M, Okuno NM, Bortolotti H, et al. Improving cycling performance: transcranial direct current stimulation increases time to exhaustion in cycling. PloS one. 2015;10(12):e0144916.

12. Williams PS, Hoffman RL, Clark BC. Preliminary evidence that anodal transcranial direct current stimulation enhances time to task failure of a sustained submaximal contraction. PloS one. 2013;8(12):e81418.

13. Sasada S, Endoh T, Ishii T, Komiyama T. Polarity-dependent improvement of maximal-effort sprint cycling performance by direct current stimulation of the central nervous system. Neuroscience Letters. 2017;657:97-101.

14. Okano AH, Machado DGS, Neto LO, et al. Can Transcranial Direct Current Stimulation Modulate Psychophysiological Response in Sedentary Men during Vigorous Aerobic Exercise? International Journal of Sports Medicine. 2017;38(07):493-500.

15. Karnath H-O, Baier B, Nägele T. Awareness of the functioning of one's own limbs mediated by the insular cortex? Journal of Neuroscience. 2005;25(31):7134-7138.

16. Barwood MJ, Butterworth J, Goodall S, et al. The effects of direct current stimulation on exercise performance, pacing and perception in temperate and hot environments. Brain Stimulation. 2016;9(6):842-849. 
17. Bar-Or O. The Wingate anaerobic test an update on methodology, reliability and validity. Sports Medicine. 1987;4(6):381-394.

18. Thompson WR, Gordon NF, Pescatello LS. ACSM's Guidelines for Exercise Testing and Prescription. 8th ed. Philadelphia (PA): Lippincott Williams \& Wilkins; 2009.

19. Borg GA. Perceived exertion as an indicator of somatic stress. Scandinavian Journal of Rehabilitation Medicine. 1970;2(2):92.

20. Klem GH, LuÈders HO, Jasper H, Elger C. The ten-twenty electrode system of the International Federation. Electroencephalogr Clin Neurophysiol. 1999;52(3):3-6.

21. Gandiga PC, Hummel FC, Cohen LG. Transcranial DC stimulation (tDCS): a tool for double-blind shamcontrolled clinical studies in brain stimulation. Clinical Neurophysiology. 2006;117(4):845-850.

22. Jaafar H, Rouis M, Coudrat L, Attiogbé E, Vandewalle H, Driss T. Effects of load on Wingate test performances and reliability. The Journal of Strength \& Conditioning Research. 2014;28(12):3462-3468.

23. Cohen J. Statistical power analysis for the behavioral sciences 2nd edn. In: Erlbaum Associates, Hillsdale; 1988.

24. Lattari E, Oliveira BR, Junior RSM, et al. Acute effects of single dose transcranial direct current stimulation on muscle strength: A systematic review and meta-analysis. PloS one. 2018;13(12).

25. Flood A, Waddington G, Keegan RJ, Thompson KG, Cathcart S. The effects of elevated pain inhibition on endurance exercise performance. PeerJ. 2017;5:e3028.

26. Robertson CV, Marino FE. A role for the prefrontal cortex in exercise tolerance and termination. Journal of Applied Physiology. 2016;120(4):464-466.

27. Machado S, Jansen P, Almeida V, Veldema J. Is tDCS an Adjunct Ergogenic Resource for Improving Muscular Strength and Endurance Performance? A Systematic Review. Frontiers in Psychology. 2019;10(1127).

28. Zupan MF, Arata AW, Dawson LH, Wile AL, Payn TL, Hannon ME. Wingate Anaerobic Test Peak Power and Anaerobic Capacity Classifications for Men and Women Intercollegiate Athletes. The Journal of Strength \& Conditioning Research. 2009;23(9):2598-2604.

29. Hunter AM, St Clair Gibson A, Lambert MI, Nobbs L, Noakes TD. Effects of supramaximal exercise on the electromyographic signal. British journal of sports medicine. 2003;37(4):296-299.

30. Lattari E, Andrade ML, Alberto Filho S, et al. Can Transcranial Direct Current Stimulation Improve the Resistance Strength and Decrease the Rating Perceived Scale in Recreational Weight-Training Experience? Journal of Strength and Conditioning Research. 2016;30(12):3381-3387.

31. Lattari E, Rosa BF, Fonseca SJ, et al. Effects on Volume Load and Ratings of Perceived Exertion in Individuals Advanced Weight-Training After Transcranial Direct Current Stimulation. Journal of Strength \& Conditioning Research. 2018.

32. Lattari E, Campos C, Lamego MK, et al. Can transcranial direct current stimulation improve muscle power in individuals with advanced resistance training experience? The Journal of Strength \& Conditioning Research. 2020;34(1):97-103.

33. Holgado D, Vadillo MA, Sanabria D. The effects of transcranial direct current stimulation on objective and subjective indexes of exercise performance: A systematic review and meta-analysis. Brain Stimulation. 2019;12(2):242-250.

34. Unal M, Unal DO. Gene doping in sports. Sports Medicine. 2004;34(6):357-362.

35. Reardon S. Performance boost paves way for'brain doping': electrical stimulation seems to boost endurance in preliminary studies. Nature. 2016;531(7594):283-285. 